\title{
Is Incoherence Surprising? Targeted Evaluation of Coherence Prediction from Language Models
}

\author{
Anne Beyer \\ Sharid Loáiciga \\ David Schlangen \\ Computational Linguistics, Department of Linguistics, University of Potsdam, Germany \\ \{anne.beyer, loaicigasanchez, david.schlangen\}@uni-potsdam.de
}

\begin{abstract}
Coherent discourse is distinguished from a mere collection of utterances by the satisfaction of a diverse set of constraints, for example choice of expression, logical relation between denoted events, and implicit compatibility with world-knowledge. Do neural language models encode such constraints? We design an extendable set of test suites addressing different aspects of discourse and dialogue coherence. Unlike most previous coherence evaluation studies, we address specific linguistic devices beyond sentence order perturbations, allowing for a more fine-grained analysis of what constitutes coherence and what neural models trained on a language modelling objective do encode. Extending the targeted evaluation paradigm for neural language models (Marvin and Linzen, 2018) to phenomena beyond syntax, we show that this paradigm is equally suited to evaluate linguistic qualities that contribute to the notion of coherence.
\end{abstract}

\section{Introduction}

Statistical models trained on large amounts of data using the language modelling objective (predicting words in context) have shown to pick up an intriguing amount of implicit knowledge about other tasks, for example syntactic knowledge (Warstadt et al., 2020; Hu et al., 2020) or world knowledge (Trinh and Le, 2019; Tamborrino et al., 2020). They have also been shown to exhibit, within these tasks, interesting divergences from expectation and sensitivity to confounding factors (e.g. McCoy et al. (2019)).

Inspired by the recently released SyntaxGym (Gauthier et al., 2020), which enables specific and standardised evaluation of syntactic knowledge encoded in such models, we explore whether similar methods can be applied to the study of discourse knowledge or coherence, i.e., constraints acting across sentence boundaries, as illustrated in (1) (where "\#" marks the less acceptable variant).
a. \#The lone ranger rode off into the sun- set. Then he jumped on his horse.
b. The lone ranger jumped on his horse. Then he rode into the sunset.

A common approach to coherence evaluation consists in shuffling the sentence order of a text, thereby creating incoherent text samples that need to be discriminated from the original (Barzilay and Lapata, 2008). While this approach to creating incoherent test data is intuitive enough, recent studies suggest that it paints only a partial picture of what constitutes coherence (Lai and Tetreault, 2018; Mohammadi et al., 2020; Pishdad et al., 2020). It does not pinpoint the qualities that make the shuffled text incoherent, it does not tell us which linguistic devices are at fault, emphasising the need to move beyond this technique. This paper aims to add to the growing body of research stressing the need for more qualitative evaluations of text coherence (See et al., 2019; Mohammadi et al., 2020; Pishdad et al., 2020).

We design different test suites created semiautomatically from existing corpora. This eases the burden of creating them from scratch and ensures the inclusion of multiple genres, crucially including dialogue data. Each test suite addresses a hypothesis about an underlying linguistic device contributing to a text's coherence, i.e., choice of referring expressions, discourse connectives, and intention (speaker commitment).

Our contributions are the following: We

- extend SyntaxGym to handle phenomena acting across sentence boundaries, but keep the general functionality to allow the use of both syntactic and coherence test suites,

- show that it is possible to evaluate dialogue models by extending $l \mathrm{~m}-z \circ 0$ (SyntaxGym's model repository), and

- present a first set of coherence test suites, each 
assessing a fine-grained and linguistically motivated element of coherence.

Our work thus eliminates the need for adapting and gathering various benchmark datasets by providing an easily extensible coherence evaluation framework that allows the use of existing test suites and the design of new ones. At the moment, all of the test suites reported below are in English, but we come back to possible extensions in Section 5 .

Our results are mixed: To the extent that the test suites effectively capture coherence, the examined models are neither systematically incoherent nor coherent. We take this as support for our claim that more and better linguistically informed test suites are needed in oder to fully understand if neural models actually do capture genuine coherence. We expect to develop our work further, but at this point, our contribution is a systematic framework that will allow us to do just that.

The code to create our test suites can be found at https://github.com/AnneBeyer/ coherencegym.

\section{Related Work}

SyntaxGym. Gauthier et al. (2020) develop a toolkit for targeted evaluation of language models on different syntactic phenomena. It is built on top of $l \mathrm{~m}-\mathrm{z} \circ \mathrm{O}^{1}$ a repository of language models that each specify their corresponding function to extract token level surprisal values $s(t)$ from the language model's conditional token probabilities $p$.

$$
s\left(t_{i}\right)=-\log _{2}\left(p\left(t_{i} \mid t_{0} \ldots t_{i-1}\right)\right)
$$

Different syntactic phenomena can be evaluated by running models on different test suites. Each test suite contains items with minimally different conditions, focusing on the specific phenomenon. An example item for NUMBER AGREEMENT is given below.

a. condition name: match

region 1: The woman

region 2: plays

region 3: the guitar

b. condition name: mismatch

region 1: The woman

region 2: play

region 3: the guitar

\footnotetext{
${ }^{1}$ https://cpllab.github.io/lm-zoo/
}

Each test suite also contains a prediction of the expected difference between conditions. Splitting the input into different regions makes it possible to measure the difference in model predictions at the token or phrase level. (e.g. region 2 in condition mismatch should be more surprising than region 2 in condition match).

Coherence. While the notion of syntactic acceptability is well studied from a linguistic point of view and in terms of neural language model representations (Marvin and Linzen, 2018; Warstadt et al., 2019, 2020; Hu et al., 2020, inter alia), it remains less clear what neural models are capable of capturing when modelling language across sentence boundaries.

There exists a large body of work in linguistics regarding different notions of coherence, such as the influence of coreference (Hobbs, 1979; Barzilay and Lapata, 2008, inter alia), Centering theory (Grosz et al., 1995), discourse structure (Mann and Thompson, 1987; Webber et al., 2003), and phenomena that connect utterances in dialogue, such as conversational maxims (Grice, 1975) or speaker interaction (Lascarides and Asher, 2009).

Many of these are also mentioned by coherence evaluation studies, nonetheless they mostly revert to the use of some form of sentence-order variations (Chen et al., 2019; Moon et al., 2019; Xu et al., 2019; Mesgar et al., 2020). While some progress has been made towards incorporating more linguistically motivated test sets (Chen et al., 2019; Mohammadi et al., 2020; Pishdad et al., 2020), most evaluation studies focus on models trained specifically on coherence classification and prediction tasks.

Language models. The recently proposed transformer language model GPT-2 (Radford et al., 2019) has been shown to perform very well on many downstream language tasks. See et al. (2019) quantitatively evaluate GPT-2 as a language generator and find that it generally performs on par with a state-of-the-art neural story generation model. However, they also note that their automatic measures focus mostly on text diversity and stress the need for more qualitative evaluation methods for notions like text coherence.

GPT-2 is also the basis of the recently proposed dialogue model DiALOGPT (Zhang et al., 2020), which is fine-tuned on conversational data from Reddit. Mehri and Eskenazi (2020) argue that DI- 
ALOGPT encodes several notions of dialogue quality, including coherence. They manually create several positive and negative follow-up utterances for certain dialog qualities (e.g. "Wow, that's interesting!" or "I'm confused."). The likelihood of DIALOGPT outputting either of them is then used to give an overall score per quality. The notion of dialogue coherence, although shown to be among the most important for predicting overall dialogue quality, is found to be one of the hardest to predict using this method. The authors attribute this to the fact that coherence (or the lack thereof) is seldom verbalised, so the model is not able to associate this notion with specific follow-up utterances. We take this a step back and evaluate the evaluator in order to get a better understanding of which notions of coherence are actually implicitly encoded in DialoGPT.

We test GPT-2 and DiALOGPT on different notions of discourse and dialogue coherence by evaluating them on specifically designed test suites building on the SyntaxGym methodology.

\section{From SyntaxGym to CoherenceGym: Querying Coherence Judgements and Creating Datasets}

We show that the methods implemented in SyntaxGym can also be applied to evaluate phenomena that go beyond a single sentence. SyntaxGym is based on the psycholinguistically motivated notion of surprisal, which they utilise to compare the scores assigned by a language model to specific regions in a minimal pair of sentences. In our CoherenceGym setting, the regions of interest comprise larger chunks up to whole sentences. We calculate the models' token level surprisals and aggregate them over all tokens $t_{1} \ldots t_{n}$ in the region $r$ of interest. As the continuations may differ in more than one token and can be of different lengths, we use the mean region surprisal. ${ }^{2}$

$$
s_{\text {mean }}(r)=\frac{1}{n} \sum_{i=1}^{n} s\left(t_{i}\right)
$$

To create incoherent versions, we utilise several existing datasets and devise different modifications that target a concrete phenomenon. We also include some existing methods and resources in order to demonstrate that those can easily be integrated and to cover a wide range of phenomena, which are

\footnotetext{
${ }^{2}$ This required a slight adaptation of syntaxgym, which is now part of the official implementation.
}

described in detail in Section 4. We further add DiALOGPT (Zhang et al., 2020) to the $1 \mathrm{~m}-z \circ 0$ to show that the coherence test suites can also be used to evaluate dialogue models. ${ }^{3}$

The Coherence Detection (CD) scores reported in Section 4 measure the proportion of items for which each model met the prediction of each test suite, i.e., the prediction accuracy of whether the model found the incoherent version more surprising than the coherent counterpart.

\subsection{Models}

SyntaxGym is built as a wrapper on top of $\mathrm{lm}-\mathrm{z} 0 \mathrm{O}$, a repository of language model Docker containers specifying the functions tokenizer, unkify and get_surprisals. GPT-2 (117M) (Radford et al., 2019) is already included by the developers, based on the huggingface transformers library. ${ }^{4}$ We use this version and add DIALOGPT (Zhang et al., 2020), which is built upon GPT-2, but further fine-tuned on Reddit data, in the same manner. As Reddit contains multi-person dialogues, the separator token is taken to denote speaker change. Both models compute the next token probability based on the softmax output of the final linear layer of the decoder. Following the get_surprisals function for GPT-2, we transform the token probabilities into surprisals as shown in Equation 1.

Each of the two models exist in different versions, depending on the number of parameters (embedding size, number of layers). For technical reasons, we used the small version of GPT-2 (117M) and the medium version of DIALOGPT(345M), so the two models are not directly comparable. As the aim of this study is to show that the surprisal based targeted evaluation paradigm is useful for coherence evaluation in general, we leave a detailed comparison of the impact of different model sizes to future work.

\section{Coherence Phenomena and Test Suites}

In this section, we describe the different coherence phenomena assessed by our test suites. For every test suite we first posit a hypothesis, which is coded into the suite's prediction section. Next, we describe the dataset and the manipulation applied

\footnotetext{
${ }^{3}$ This implies some restrictions on compatibility though: All models should be able to predict discourse coherence phenomena, but only dialogue models need to additionally encode dialogue coherence.

${ }^{4}$ https://huggingface.co/transformers/
} 
to create incoherent samples that exhibit a violation of coherence regarding the specific phenomenon. Each subsection reports the results of the evaluated models on the respective test suite. As we evaluate models pre-trained on English data, our test suites are devised only in English as well.

The first three test suites are based on existing methods or test sets that we integrate into the framework. The following three test suites are newly created.

\subsection{Sentence Order Baseline Test Suite}

Hypothesis: A coherent text is composed of an ordered set of sentences in a logical sequence; shuffling the sentences breaks the logical order and hence coherence. Since sequentiality is central to the language modelling task, models successfully distinguish between both versions.

This shuffling technique has been widely applied in the evaluation of coherence models (Barzilay and Lapata, 2008; Chen et al., 2019; Moon et al., 2019; Xu et al., 2019; Mesgar et al., 2020). We include it as baseline for our method, in order to contrast how more fine-grained notions of coherence compare to this broad approach.

We use ROCStories (Mostafazadeh et al., 2016) and the PERsona-ChAT corpus (Zhang et al., 2018) to evaluate sentence order for narration as well as dialogue data. The ROCStories corpus consists of coherent five-sentence stories which were gathered by employing crowdworkers and contain several temporal and causal relations between the sentences. To create the PERSONA-ChAT corpus (Zhang et al., 2018), crowd sourced dialogue participants were assigned a persona in the form of descriptive natural language sentences and were asked to talk to each other impersonating their assigned persona. The dialogues contain at least 6 turns and we extract only the utterances and ignore the persona descriptions.

Two versions are created of both corpora:

1. We shuffle all utterances and compare the aggregated overall surprisal for all tokens over all regions.

2. We keep the last utterance fixed and shuffle only the context and compare the aggregated surprisal for the second region (cf. (3)).

\section{a. condition name: original}

region 1: My friends all love to go to

the club to dance. They think it's a

\begin{tabular}{|c|c|c|c|c|}
\hline & N_all & N_context & D_all & D_context \\
\hline GPT-2 & 0.86 & 0.50 & 0.96 & 0.72 \\
\hline DIALOGPT & 0.78 & 0.44 & 0.86 & 0.55 \\
\hline \#items & 1871 & 1871 & 967 & 967 \\
\hline
\end{tabular}

Table 1: CD scores on shuffling test suites. $(\mathrm{N}=$ narration, $\mathrm{D}=$ dialogue data, all refers to the shuffling of all sentences, context is based on comparing the surprisals of the last sentence with ordered or shuffled context.

lot of fun and always invite. I finally decided to tag along last Saturday. I danced terribly and broke a friend's toe.

region 2: The next weekend, I was asked to please stay home.

b. condition name: shuffled

region 1: I finally decided to tag along last Saturday. I danced terribly and broke a friend's toe. My friends all love to go to the club to dance. They think it's a lot of fun and always invite. region 2: The next weekend, I was asked to please stay home.

Results. As Table 1 shows, shuffling is a good first indicator for detecting coherence on a global level, as the models perform quite well in the conditions where all sentences have been shuffled. ${ }^{5}$

On a local level (i.e., the influence that shuffling the context has on the following sentence), however, the ability to detect the manipulated sequence drops largely, even to or below chance. A manual inspection of the data in the context condition revealed that, in some cases, the final (non-moved) utterance (region 2) also can be judged as a coherent follow-up to the utterance shuffled into the final context position. This also reveals that shuffling does not always break coherence in the expected way due to the nature of natural language, thus highlighting the importance of a more thoughtful design of coherence test suites.

\subsection{Story Cloze Test Suite}

Hypothesis: Combining commonsense and discourse relations enables a model to detect a co-

\footnotetext{
${ }^{5}$ It is worth noting that by fine-tuning on user generated content, this ability decreases, which probably says more about Reddit than aboutDIALOGPT, but as noted before, these results are not directly comparable as the models are of different sizes.
} 
herent from an incoherent ending of a given story. We use the same corpus as for the narration shuffling condition above, but keep the order intact. The Story Cloze test set (Mostafazadeh et al., 2016) contains an additional implausible ending to each story. We use the annotated test set of the spring 2016 version and create items with different endings as exemplified in (4).

\section{a. condition name: original ending} region 1: My friends all love to go to the club to dance. They think it's a lot of fun and always invite. I finally decided to tag along last Saturday. I danced terribly and broke a friend's toe.

region 2: The next weekend, I was asked to please stay home.

b. condition name: distractor ending region 1: My friends all love to go to the club to dance. They think it's a lot of fun and always invite. I finally decided to tag along last Saturday. I danced terribly and broke a friend's toe.

region 2: My friends decided to keep inviting me out as I am so much fun.

Calculating our CD score allows for a direct evaluation of language models without the need for training a classifier on top of the model representations.

Results. The first column in Table 2 displays the results on the Story Cloze test suite. While these results leave room for improvement, it is worth noting that they are on par or even outperform the models from the original paper, which mostly rely on semantic similarities between the context and the continuations. However, we still do not learn which linguistic devices are responsible for the perception of coherence or incoherence of a given ending from this data. The following test suites are designed to investigate specific phenomena of coherence and models abilities to encode them in more detail.

\subsection{Winograd Schema Test Suite}

Hypothesis: Models are able to combine commonsense knowledge with pronoun resolution, thus they are able to distinguish the correct target from the distractor in Winograd Schema style sentences. This dataset was proposed by Trinh and Le (2019)

\begin{tabular}{lccc}
\hline & Story Cloze & \multicolumn{2}{c}{ Winograd } \\
& & full & partial \\
\hline GPT-2 & 0.61 & 0.53 & 0.59 \\
\hline DiALOGPT & 0.57 & 0.55 & 0.57 \\
\hline \#items & 1871 & 273 & 273 \\
\hline
\end{tabular}

Table 2: CD scores on the Story Cloze and the Winograd test suites (full is based on comparing the surprisals of the whole sequences, partial only considers the regions following the inserted referent)

as has also been applied by Radford et al. (2019) for evaluating GPT-2's commonsense knowledge. We reproduce the test suite in the following way:

\section{a. condition name: target}

region 1: The city councilmen refused the demonstrators a permit because region 2 : the city councilmen region 3: feared violence.

b. condition name: distractor region 1: The city councilmen refused the demonstrators a permit because region 2 : the demonstrators region 3: feared violence.

Following Trinh and Le (2019) and Radford et al. (2019), we compare the full version (comparing the mean surprisal over all tokens) and a partial version (comparing the surprisal for region 3).

Results. The last two columns in Table 2 report the CD scores for the Winograd test suite.

As noted by Trinh and Le (2019), the difference in language model scores is more obvious in the region following the inserted correct or distracting entity. We are able to reproduce these results in our setting, which supports the applicability of the CoherenceGym approach. Radford et al. (2019) demonstrate that the performance on this task can be increased by adding more parameters to the model. We will inspect the impact of model sizes on the different test suites more closely in future work.

\subsection{Coreference Test Suite}

Hypothesis: Different referring expressions reflect both the accessibility and salience status of the entities being referred. For keeping in topic however, entities need only to be re-mentioned, regardless of their form. In this sense, language models are 
insensitive to the use of different referring expressions.

In line with theories proposing an accessibility hierarchy that position pronouns requiring the highest level of accessibility and lexical noun phrases (undefinites and definites) the lowest level (Givón, 1983; Ariel, 2004, cf.), we test whether language models capture a violation in the use of referring expressions according to their accessibility status.

For this test suite, we work with the ARRAU corpus (Uryupina et al., 2020). In contrast to other coreference corpora, ARRAU is multi-genre -including news, dialogue and fiction texts- and provides annotations for non-nominal anaphora such as discourse deixis.

We extract coreferential chains whose mentions span consecutive sentences and with at least one pronominal mention. The test suites examples consist of minimal pairs (6) where a same context sentence in region 1 containing the antecedent is followed by the sentence with the original pronoun re-mentioning the antecedent or by a manipulated sentence in which the pronoun is replaced by a repetition of the antecedent in region 2.

a. condition name: pronoun

region 1: And there's a ladder coming out of the tree and there's a man at the top of the ladder

region 2: you can't see him yet

b. condition name: repetition

region 1: And there's a ladder coming out of the tree and there's a man at the top of the ladder

region 2: you can't see the man at the top of the ladder yet

In keeping with the accessibility theory, we have replaced the indefinite marker $a$ with a definite the in the repetition condition.

Results. The results show that when presented with a new lexical entity, neither model has a clear preference for a pronominal re-mention of the entity (Table 3 ). The very nature of the language model will drive it to topic continuity, as it is designed to generate tokens based on a previous history. However, this does not automatically ensures cohesion. Both pronominalisation and repetition represent cohesive ties to the previous context recoverable from surface cues. The difference is that the first involves a stronger link with the context, licensing the use of the pronoun, which the models

\begin{tabular}{lcccc}
\hline & wSJ & vPC & Dialogue & Fiction \\
\hline GPT-2 & 0.53 & 0.56 & 0.47 & 0.42 \\
\hline DiALOGPT & 0.44 & 0.51 & 0.47 & 0.36 \\
\hline \#items & 512 & 75 & 68 & 98 \\
\hline
\end{tabular}

Table 3: CD score results on entity re-mention test suite. WSJ and VPC refer to the News portion of the ARRAU corpus.

evaluated here fail to pick up.

\subsection{Explicit Connectives Test Suite}

Hypothesis: Meaning is constructed by building a representation for each new sentence based on the content of the previous sentences, and a first level of the coherence between two segments is embodied by explicit connectives. Hence, an inappropriate connective between two segments will yield a content gap. Sensitivity to content-meaning implies then sensitivity to a change in explicit connectives. For this exercise, we work with Disco-Annotation (Popescu-Belis et al., 2012), a corpus of segments from the Europarl corpus (Koehn, 2005) annotated with discourse connective senses. ${ }^{6}$ Eight discourse connectives are annotated in the corpus (as, although, though, while, since, yet, however, meanwhile), with one of five possible senses (contrast, concession, causal, temporal, comparison). We excluded all examples where the connective is in a segment initial position, since the previous segment is not provided, a setting incompatible with our constraints. This removed all examples of meanwhile. A minimal pair is created from each segment (7), where all the tokens up to the connective are used as context, followed by the original connective or another connective from the set, and the continuation of the segment.

a. condition name: original

region 1: We share the widespread outrage at its attitude to history, in particular World War II, but also its policies on enlargement, on immigration, on race and its attitude to the European Union itself. We were also outraged, region 2: however region 3: , at the tolerance of the left

\footnotetext{
${ }^{6}$ Europarl segments are either very long sentences formed by several clauses or by $2-3$ sentences clustered together, as a product of the sentence alignment process.
} 
for the tyranny, the terror and the excesses of the former USSR.

\section{b. condition name: manipulated}

region 1: We share the widespread outrage at its attitude to history, in particular World War II, but also its policies on enlargement, on immigration, on race and its attitude to the European Union itself. We were also outraged, region 2: since

region 3: , at the tolerance of the left for the tyranny, the terror and the excesses of the former USSR.

Some connectives may have the same sense depending on the specific context in which they appear (Stede, 2012; Webber et al., 2003), for instance both since and while may bear a temporal interpretation. On that account, we expect that a replacement with a different connective bearing a different sense leads to region 3 being more surprising than a different connective able to have the same sense.

Results. Not all relations captured by the connectives are equally difficult, producing high variability in the scores, as shown in Table 4. While temporal senses seem to be relatively unproblematic (scores about 0.85 on average, GPT-2), 'contrast', 'concession' and in particular 'causal' senses are more difficult to distinguish (since_causal and as_causal have averages of 0.66 and 0.52 respectively).

The results for as present an interesting contrast. This connective can also be used as a preposition. When the connectives with this particular sense are replaced, the models do not have any trouble recognising the original from the manipulated sentence, as suggested by the systematic high scores obtained, between 0.96 and 0.99 . In most other senses, however, scores plummet as low as 0.28. We observe a similar pattern for yet when used as an adverb in the DIALOGPT model.

\subsection{Speaker Commitment Test Suite}

Hypothesis: While it is possible for different speakers to have different opinions, speakers should not contradict themselves. This test suite targets the notion of speaker commitment in dialogue models. The test suite is created automatically based on the DialogueNLI corpus (Welleck et al., 2019), which contains pairs of utterances annotated as contradiction, entailment or neutral. The sentence pairs are extracted from the PERSONA-CHAT corpus introduced in Section 4.1. The sentences can either be part of the conversation or the persona descriptions. We extract the contradicting sentence pairs from the human verified test set, and create two conditions for each utterance pair, as illustrated below:

a. condition name: speaker change region 1: since the beginning of the year, $i$ am a nurse. [SEP] region 2: i am a kindergarten teacher.

b. condition name: same speaker region 1: since the beginning of the year, $i$ am a nurse.

region 2: i am a kindergarten teacher.

In the first condition, we simulate a speaker change by introducing a $[S E P]$ token (which is converted to the tokenizer's separator token internally) in the dialogue history, whereas in the second condition the continuation is uttered by the same speaker as the context.

A model that is encoding some notion of speaker commitment should find the second utterance more surprising if no speaker change occurred.

As non-dialogue language models do not encode the notion of speaker change, this test suite only yields relevant results for dialogue models.

Results. DialoGPT shows a tendency towards finding contradictions within the same speaker more surprising. A manual inspection of the data revealed that even though we use the human verified test set, there are quite some instances where the implications are not as clear, for example in the following two sentence pairs:

$$
\begin{aligned}
& \text { a. } \quad \text { "my nurse skills come in handy when } \\
& \text { i volunteer." } \\
& \text { "i am a kindergarten teacher." } \\
& \text { b. } \quad \text { "i love art and want to be a famous } \\
& \text { artist." } \\
& \text { "i am a kindergarten teacher." }
\end{aligned}
$$

This highlights the importance of quality over quantity. In future work, we will inspect this phenomenon more closely and combine the selection of items with human evaluation, to gain a better understanding of how the notion of speaker commitment is and can be encoded in neural dialogue models. 


\begin{tabular}{|c|c|c|c|c|c|c|c|c|c|c|c|c|c|c|}
\hline \multirow{4}{*}{$\begin{array}{l}\text { CONNECTIVE SENSE } \\
\text { although_concession } \\
\text { although_contrast }\end{array}$} & \multicolumn{7}{|c|}{$\begin{array}{c}\text { GPT-2 } \\
\text { Connective used in manipulation }\end{array}$} & \multicolumn{7}{|c|}{$\begin{array}{c}\text { DIALOGPT } \\
\text { Connective used in manipulation }\end{array}$} \\
\hline & although & as & however & since & though & while & yet & although & as & however & since & though & while & yet \\
\hline & - & 0.92 & 0.92 & 0.857 & 0.84 & 0.86 & 0.90 & - & 0.88 & 0.83 & 0.82 & 0.76 & 0.76 & 0.89 \\
\hline & - & 1.00 & 0.86 & 0.86 & 0.43 & 1.00 & 0.86 & - & 1.00 & 0.86 & 1.00 & 0.93 & 0.79 & 1.00 \\
\hline as_causal & 0.44 & - & 0.80 & 0.28 & 0.64 & 0.72 & 0.80 & 0.64 & - & 0.68 & 0.40 & 0.84 & 0.80 & 0.88 \\
\hline as_comparison & 0.96 & - & 0.95 & 0.96 & 0.94 & 0.97 & 0.97 & 0.86 & - & 0.89 & 0.86 & 0.93 & 0.87 & 0.92 \\
\hline as_concession & 0.33 & - & 0.67 & 0.67 & 0.33 & 1.00 & 1.00 & 0.67 & - & 0.67 & 0.67 & 1.00 & 1.00 & 0.67 \\
\hline as_PREPOSITION & 0.99 & - & 0.99 & 0.98 & 0.99 & 0.99 & 0.99 & 0.96 & - & 0.97 & 0.97 & 0.97 & 0.97 & 0.98 \\
\hline as_temporal & 0.95 & - & 0.95 & 0.86 & 1.00 & 0.81 & 0.95 & 0.86 & - & 1.00 & 0.86 & 1.00 & 0.76 & 1.00 \\
\hline however_concession & 0.70 & 0.90 & - & 0.86 & 0.63 & 0.80 & 0.64 & 0.58 & 0.79 & - & 0.79 & 0.71 & 0.71 & 0.53 \\
\hline however_contrast & 0.67 & 0.89 & - & 0.89 & 0.33 & 0.89 & 0.67 & 0.67 & 0.89 & - & 0.78 & 0.56 & 0.67 & 0.56 \\
\hline since_causal & 0.61 & 0.78 & 0.83 & - & 0.72 & 0.79 & 0.93 & 0.66 & 0.82 & 0.74 & - & 0.83 & 0.79 & 0.89 \\
\hline since_temporal-causal & 1.00 & 0.83 & 1.00 & - & 1.00 & 1.00 & 1.00 & 0.67 & 1.00 & 1.00 & - & 0.83 & 0.83 & 1.00 \\
\hline since_temporal & 0.96 & 0.97 & 0.96 & - & 0.94 & 0.97 & 0.98 & 0.95 & 0.97 & 0.95 & - & 0.95 & 0.92 & 0.95 \\
\hline though_concession & 0.43 & 0.82 & 0.79 & 0.87 & - & 0.78 & 0.90 & 0.37 & 0.87 & 0.76 & 0.82 & - & 0.78 & 0.79 \\
\hline though_contrast & 0.56 & 0.84 & 0.84 & 0.88 & - & 0.88 & 0.80 & 0.41 & 0.75 & 0.59 & 0.77 & - & 0.72 & 0.83 \\
\hline while_concession & 0.46 & 1.00 & 1.00 & 0.96 & 0.78 & - & 0.98 & 0.57 & 0.96 & 0.87 & 0.89 & 0.76 & - & 0.93 \\
\hline while_contrast & 0.78 & 0.93 & 0.93 & 0.85 & 0.81 & - & 0.81 & 0.81 & 0.93 & 0.81 & 0.85 & 0.96 & - & 0.81 \\
\hline while_temporal-causal & 0.90 & 0.80 & 0.90 & 0.70 & 0.80 & - & 0.80 & 0.80 & 0.80 & 1.00 & 0.70 & 0.90 & - & 1.00 \\
\hline while_temporal-contrast & 0.73 & 0.90 & 0.90 & 0.73 & 0.77 & - & 0.81 & 0.67 & 0.81 & 0.81 & 0.85 & 0.88 & - & 0.81 \\
\hline while_temporal & 0.57 & 0.57 & 0.71 & 0.86 & 0.71 & - & 0.86 & 0.86 & 0.71 & 0.86 & 0.86 & 1.00 & - & 1.00 \\
\hline yet_ADV & 0.95 & 0.98 & 0.82 & 0.93 & 0.93 & 0.98 & - & 0.92 & 0.96 & 0.85 & 0.92 & 0.94 & 0.97 & - \\
\hline yet_concession & 0.92 & 0.95 & 0.95 & 0.92 & 0.97 & 0.95 & - & 0.59 & 0.90 & 0.72 & 0.79 & 0.74 & 0.85 & - \\
\hline yet_contrast & 0.92 & 0.88 & 0.88 & 0.92 & 0.92 & 0.79 & - & 0.67 & 0.96 & 0.75 & 0.88 & 0.79 & 0.88 & - \\
\hline
\end{tabular}

Table 4: CD scores on explicit connectives test suite. The first column list all the connective senses from DiscoAnnotation. Scores below 0.50 are boldfaced, while the PREPOSITION and ADVERB senses are highlighted in yellow.

\begin{tabular}{lc}
\hline & contradiction \\
\hline DiALOGPT & 0.59 \\
\hline \#items & 4041 \\
\hline
\end{tabular}

Table 5: CD score for speaker commitment test suite.

\section{Conclusions}

We revisit the targeted evaluation paradigm and create test suites focusing on specific coherence phenomena. Each test suite contains minimal pairs of sequences that illustrate a specific component of coherence.

We evaluate two transformer models for language and dialogue modelling based on the token level surprisal scores they assign to the coherent and incoherent versions. Extending the existing SyntaxGym toolkit, we evaluate GPT-2 and DIALOGPT on our newly designed test suites on entity re-mention, explicit discourse connectives and speaker commitment in dialogue. Existing test sets are also integrated easily, which we demonstrate for sentence order detection, Story Cloze and Winograd Schema resolution tasks. Our results support previous work suggesting that the notion of coherence encoded in neural language models is more nuanced than the sentence order discrimination task can reflect.

The mixed results we get, with some manipulations (e.g. the different sense connective substitutions) easily being spotted by the tested models and others (e.g. how to re-mention entities, or speaker contradictions) posing to be more difficult, point to the value of such targeted evaluation, which eventually might help in pointing towards where the introduction of different inductive biases could increase a model's performance.

In this study, we focus on the English language. However, our approach is not inherently designed for English alone. While $1 \mathrm{~m}-\mathrm{zoO}$ only contains English language models at the moment, other language models can be added easily. The shuffling perturbations can be applied to any corpus. Our other test suites are based on available annotated corpora, which require some familiarity with the language, but can in principle be applied in a similar fashion to resources in other languages, such as the Potsdam Commentary Corpus (Bourgonje and Stede, 2020) for German connectives, for example. We leave a multilingual extension of our framework for future work.

Our next efforts will focus on adding more language and dialogue models to determine the impact of different model architectures and sizes. Building additional test suites in order to capture a more thor- 
ough notion of coherence is also among our priorities. Last, we plan to collect human judgements to evaluate our coherence manipulations more closely and to create an upper bound for what we can expect from neural models.

\section{Acknowledgements}

We thank Johann Seltmann and Jon Gauthier for their help with augmenting $1 \mathrm{~m}-\mathrm{zoO}$ and syntaxgym. We also thank the anonymous reviewers for their valuable feedback. This work was funded by the German Research Foundation (Deutsche Forschungsgemeinschaft, DFG) Project ID 317633480 - SFB 1287.

\section{References}

Mira Ariel. 2004. Accessibility marking: Discourse functions, discourse profiles, and processing cues. Discourse Processes, 37(2):91-116.

Regina Barzilay and Mirella Lapata. 2008. Modeling Local Coherence: An Entity-Based Approach. Computational Linguistics, 34(1):1-34.

Peter Bourgonje and Manfred Stede. 2020. The Potsdam commentary corpus 2.2: Extending annotations for shallow discourse parsing. In Proceedings of the 12th Language Resources and Evaluation Conference, pages 1061-1066, Marseille, France. European Language Resources Association.

Mingda Chen, Zewei Chu, and Kevin Gimpel. 2019. Evaluation benchmarks and learning criteria for discourse-aware sentence representations. In Proceedings of the 2019 Conference on Empirical Methods in Natural Language Processing and the 9th International Joint Conference on Natural Language Processing (EMNLP-IJCNLP), pages 649-662, Hong Kong, China. Association for Computational Linguistics.

Jon Gauthier, Jennifer Hu, Ethan Wilcox, Peng Qian, and Roger Levy. 2020. SyntaxGym: An online platform for targeted evaluation of language models. In Proceedings of the 58th Annual Meeting of the Association for Computational Linguistics: System Demonstrations, pages 70-76, Online. Association for Computational Linguistics.

Thomas Givón. 1983. Topic Continuity in Discourse: A Quantitative Cross-Language Study. John Benjamin, Amsterdam.

Herbert P Grice. 1975. Logic and conversation. In Speech acts, pages 41-58. Brill.

Barbara Grosz, Aravind K. Joshi, and Scott Weinstein. 1995. Centering: A framework for modelling the local coherence of discourse. Computational Linguistics, 21(2):203-225.
Jerry R Hobbs. 1979. Coherence and Coreference. Cognitive Science, 3(1):67-90.

Jennifer $\mathrm{Hu}$, Jon Gauthier, Peng Qian, Ethan Wilcox, and Roger Levy. 2020. A systematic assessment of syntactic generalization in neural language models. In Proceedings of the 58th Annual Meeting of the Association for Computational Linguistics, pages 1725-1744, Online. Association for Computational Linguistics.

Philipp Koehn. 2005. Europarl: A parallel corpus for statistical machine translation. In Proceedings of the 10th Machine Translation Summit, MT Summit X, pages 79-86, Phuket, Thailand.

Alice Lai and Joel Tetreault. 2018. Discourse coherence in the wild: A dataset, evaluation and methods. In Proceedings of the 19th Annual SIGdial Meeting on Discourse and Dialogue, pages 214-223, Melbourne, Australia. Association for Computational Linguistics.

A. Lascarides and N. Asher. 2009. Agreement, Disputes and Commitments in Dialogue. Journal of Semantics, 26(2):109-158.

William C. Mann and Sandra A. Thompson. 1987. Rhetorical structure theory: A theory of text organization. In Livia Polanyi, editor, The Structure of Discourse. Ablex Publishing Corporation, Norwood, N.J.

Rebecca Marvin and Tal Linzen. 2018. Targeted syntactic evaluation of language models. In Proceedings of the 2018 Conference on Empirical Methods in Natural Language Processing, pages 1192-1202, Brussels, Belgium. Association for Computational Linguistics.

Tom McCoy, Ellie Pavlick, and Tal Linzen. 2019. Right for the wrong reasons: Diagnosing syntactic heuristics in natural language inference. In Proceedings of the 57th Annual Meeting of the Association for Computational Linguistics, pages 3428-3448, Florence, Italy. Association for Computational Linguistics.

Shikib Mehri and Maxine Eskenazi. 2020. Unsupervised evaluation of interactive dialog with DialoGPT. In Proceedings of the 21th Annual Meeting of the Special Interest Group on Discourse and Dialogue, pages 225-235, 1st virtual meeting. Association for Computational Linguistics.

Mohsen Mesgar, Sebastian Bücker, and Iryna Gurevych. 2020. Dialogue coherence assessment without explicit dialogue act labels. In Proceedings of the 58th Annual Meeting of the Association for Computational Linguistics, pages 1439-1450, Online. Association for Computational Linguistics.

Elham Mohammadi, Timothe Beiko, and Leila Kosseim. 2020. On the creation of a corpus for coherence evaluation of discursive units. In Proceedings of the 12th Language Resources and Evaluation Conference, pages 1067-1072, Marseille, France. European Language Resources Association. 
Han Cheol Moon, Tasnim Mohiuddin, Shafiq Joty, and Chi Xu. 2019. A unified neural coherence model. In Proceedings of the 2019 Conference on Empirical Methods in Natural Language Processing and the 9th International Joint Conference on Natural Language Processing (EMNLP-IJCNLP), pages 2262 2272, Hong Kong, China. Association for Computational Linguistics.

Nasrin Mostafazadeh, Nathanael Chambers, Xiaodong He, Devi Parikh, Dhruv Batra, Lucy Vanderwende, Pushmeet Kohli, and James Allen. 2016. A corpus and cloze evaluation for deeper understanding of commonsense stories. In Proceedings of the 2016 Conference of the North American Chapter of the Association for Computational Linguistics: Human Language Technologies, pages 839-849, San Diego, California. Association for Computational Linguistics.

Leila Pishdad, Federico Fancellu, Ran Zhang, and Afsaneh Fazly. 2020. How coherent are neural models of coherence? In Proceedings of the 28th International Conference on Computational Linguistics, pages 6126-6138, Barcelona, Spain (Online). International Committee on Computational Linguistics.

Andrei Popescu-Belis, Thomas Meyer, Jeevanthi Liyanapathirana, Bruno Cartoni, and Sandrine Zufferey. 2012. Discourse-level Annotation over Europarl for Machine Translation: Connectives and Pronouns. In Proceedings of the eighth international conference on Language Resources and Evaluation (LREC), Istanbul, Turkey.

Alec Radford, Jeff Wu, Rewon Child, David Luan, Dario Amodei, and Ilya Sutskever. 2019. Language models are unsupervised multitask learners. Technical report, OpenAI.

Abigail See, Aneesh Pappu, Rohun Saxena, Akhila Yerukola, and Christopher D. Manning. 2019. Do massively pretrained language models make better storytellers? In Proceedings of the 23rd Conference on Computational Natural Language Learning (CoNLL), pages 843-861, Hong Kong, China. Association for Computational Linguistics.

Manfred Stede. 2012. Disourse Processing. Morgan and Claypool Publishers, Toronto.

Alexandre Tamborrino, Nicola Pellicanò, Baptiste Pannier, Pascal Voitot, and Louise Naudin. 2020. Pretraining is (almost) all you need: An application to commonsense reasoning. In Proceedings of the 58th Annual Meeting of the Association for Computational Linguistics, pages 3878-3887, Online. Association for Computational Linguistics.

Trieu H. Trinh and Quoc V. Le. 2019. A Simple Method for Commonsense Reasoning. arXiv:1806.02847 [cs].

Olga Uryupina, Ron Artstein, Antonella Bristot, Federica Cavicchio, Francesca Delogu, Kepa Rodriguez, and Massimo Poesio. 2020. Annotating a broad range of anaphoric phenomena, in multiple genres: the ARRAU corpus. Natural Language Engineering, 26:95-128.

Alex Warstadt, Alicia Parrish, Haokun Liu, Anhad Mohananey, Wei Peng, Sheng-Fu Wang, and Samuel R. Bowman. 2020. BLiMP: The benchmark of linguistic minimal pairs for English. Transactions of the Association for Computational Linguistics, 8:377392.

Alex Warstadt, Amanpreet Singh, and Samuel R. Bowman. 2019. Neural network acceptability judgments. Transactions of the Association for Computational Linguistics, 7:625-641.

Bonnie Webber, Matthew Stone, Aravind Joshi, and Alistair Knott. 2003. Anaphora and discourse structure. Computational Linguistics, 29(4):545-587.

Sean Welleck, Jason Weston, Arthur Szlam, and Kyunghyun Cho. 2019. Dialogue natural language inference. In Proceedings of the 57th Annual Meeting of the Association for Computational Linguistics, pages 3731-3741, Florence, Italy. Association for Computational Linguistics.

Peng Xu, Hamidreza Saghir, Jin Sung Kang, Teng Long, Avishek Joey Bose, Yanshuai Cao, and Jackie Chi Kit Cheung. 2019. A cross-domain transferable neural coherence model. In Proceedings of the 57th Annual Meeting of the Association for Computational Linguistics, pages 678-687, Florence, Italy. Association for Computational Linguistics.

Saizheng Zhang, Emily Dinan, Jack Urbanek, Arthur Szlam, Douwe Kiela, and Jason Weston. 2018. Personalizing dialogue agents: I have a dog, do you have pets too? In Proceedings of the 56th Annual Meeting of the Association for Computational Linguistics (Volume 1: Long Papers), pages 2204-2213, Melbourne, Australia. Association for Computational Linguistics.

Yizhe Zhang, Siqi Sun, Michel Galley, Yen-Chun Chen, Chris Brockett, Xiang Gao, Jianfeng Gao, Jingjing Liu, and Bill Dolan. 2020. DIALOGPT : Large-scale generative pre-training for conversational response generation. In Proceedings of the 58th Annual Meeting of the Association for Computational Linguistics: System Demonstrations, pages 270-278, Online. Association for Computational Linguistics. 